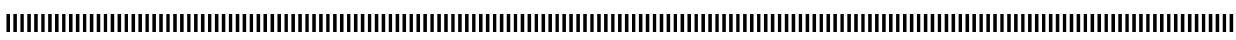

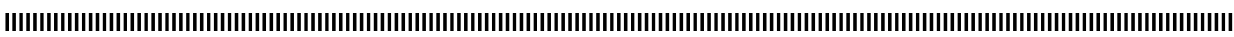

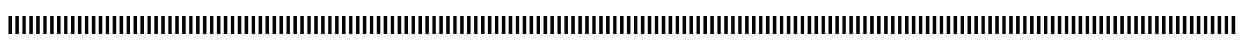

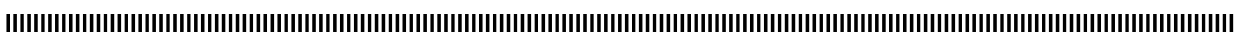

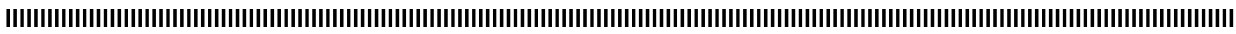

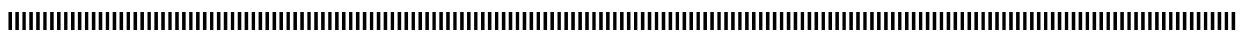

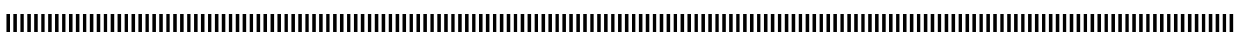

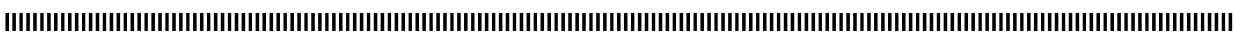

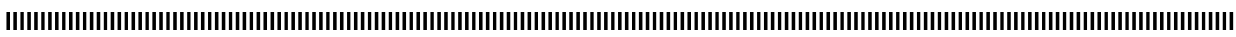

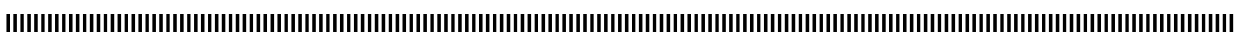

\title{
An introduction to the topological asymptotic expansion with examples
}

\author{
Fehrenbach Jérôme \\ Masmoudi Mohamed \\ Laboratoire MIP, Université Paul Sabatier \\ Toulouse, France \\ masmoudi@mip.ups-tlse.fr
}

["

ABSTRACT. To find an optimal domain is equivalent to look for its characteristic function. At first sight this problem seems to be nondifferentiable. But it is possible to derive the variation of a cost function when we switch the characteristic function from 0 to 1 or from 1 to 0 in a small area. Classical and two generalized adjoint approaches are considered in this paper. Their domain of validity is given and illustrated by several examples. Using this gradient type information, it is possible to build fast algorithms. Generally, only one iteration is needed to find the optimal shape.

RÉSUMÉ. Trouver un domaine optimal est équivalent à la recherche de sa fonction caractéristique. A première vue, ce problème semble non différentiable, mais il est possible de calculer la variation de la fonction coût lorsque la fonction caractéristique passe de 1 à 0 ou de 0 à 1 dans une région de petite taille. On s'appuyera sur une approche adjointe classique et deux généralisations de cette méthode. Le domaine de validité de ces différentes approches est donné et illustré par différents exemples. Cette information de type gradient permet de construire des algorithmes très efficaces : en général, une seule itération suffit pour trouver le domaine optimal.

KEYWORDS : Topological sensitivity, shape optimization, inverse problem, Lagrange operator, adjoint methods

MOTS-CLÉS : Sensibilité topologique, optimisation de forme, problème inverse, opérateur de Lagrange, méthodes ajointes

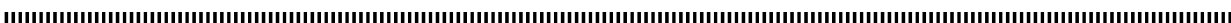

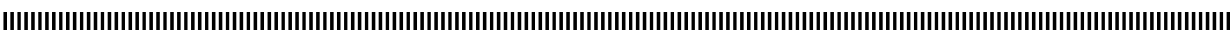

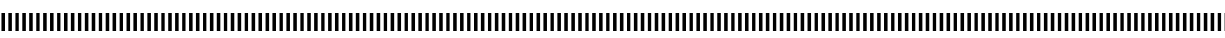

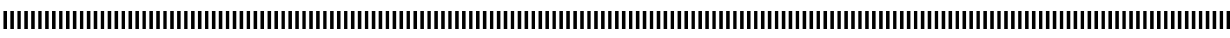

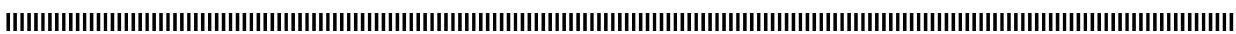

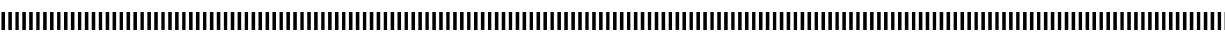

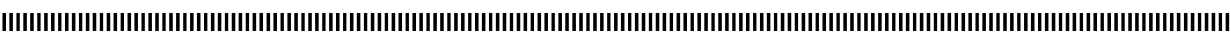
| |

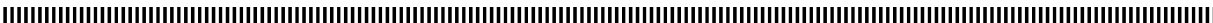




\section{Introduction}

Most of the important contributions in topological optimization concern structural mechanics and particularly the optimization of the compliance (strain potential energy) under a volume constraint. Following the idea that the optimal structure has a lot of small holes, some authors [AlK93, Ben96] use a class of composite materials. This approach leads to homogenization theory [All02]. The field of applications of these methods is quite restricted. Global optimization techniques like genetic algorithms are used in order to solve more general problems [KaS97, SKJ96]. Unfortunately these methods are very slow.

Level-set methods give interesting results [San96, AlT02]. It consists of making the boundary of the domain evolve according to a transport equation. This allows the number of holes in the domain to decrease, but not to increase.

The topological asymptotic expansion is general and efficient. As a background, we cite the contribution of Schumacher [EsS94, Sch95] under the name of bubble method in the context of compliance optimization with a Neumann boundary condition on the unknown boundary. It consists in inserting a small hole according to topological sensitivity information, then this small hole blows following classical shape optimization methods. Let us mention recent promising results, that have been obtained by coupling level-set methods with a topological asymptotic method [BHR04, WYW04, AGJ05, AmA05].

This paper is an introduction to topological asymptotic expansion methods [Ili92, SoZ99, MNP00, AVV01, GGM01, GuS01, Mas02, AVV03, SAM03, HaM04, MPS05, AHM05, Ams05]. More exactly, a shape optimization problem consists in minimizing a functional $j(\Omega)=J\left(\Omega, u_{\Omega}\right)$ where $u_{\Omega}$ is the solution to a Partial Differential Equation defined in the domain $\Omega$. Let us consider $\Omega_{\varepsilon}=\Omega \backslash B(x, \varepsilon)$ where $B(x, \varepsilon)$ is the ball of radius $\varepsilon$ about the center $x$. An asymptotic expansion of the functional $j$ can be obtained in the following form:

$$
\begin{gathered}
j\left(\Omega_{\varepsilon}\right)=j(\Omega)+f(\varepsilon) g(x)+o(f(\varepsilon)) \\
\lim _{\varepsilon \rightarrow 0} f(\varepsilon)=0, f(\varepsilon)>0 .
\end{gathered}
$$

The function $g$ is called the topological gradient.

Most of shape optimization problems could be considered via material properties optimization: we look for the distribution of a material property $c$ taking two values $c_{1}$ and $c_{2}$. If the cost function $c \mapsto \mathcal{J}(c)$ is differentiable on $L^{p}, p<2$, then the topological sensitivity $g$ is derived easily from the classical gradient of $\mathcal{J}$, it gives the variation of $\mathcal{J}$ if we switch the material property from $c_{1}$ to $c_{2}$ or from $c_{2}$ to $c_{1}$ in a small region. In section 2, we recall the classical adjoint approach. Its basic concept is to say that the gradient of the cost function is equal to the partial derivative of the Lagrange operator 
with respect to $c$ : it is not necessary to calculate the variation of the state (the solution of the direct problem) with respect to $c$.

Unfortunately, in many relevant cases from the practical point of view, the cost function is differentiable with respect to $c$, but in $L^{\infty}$. To derive a topological sensitivity, we will consider two ways to generalize the adjoint approach. In both cases, it will be necessary to take into account the variation of the state with respect to topological perturbation to obtain the variation of the cost function. This variation is known [AVV01, AVV03, AmK04, MNP00], it depends on the shape of the hole and on the boundary condition on the boundary of the hole. When the hole is simple (disc, ellipse, straight crack, ...), we obtain an explicit expansion of the solution and for more general holes, this expansion could be obtained numerically.

In section 3, we consider the first generalization technique. The existence of a Frechetlike expansion of the Lagrange operator is assumed. In particular, the leading term of the expansion is continuous with respect to state and adjoint variables. This hypothesis is satisfied if we consider a domain truncation technique around the hole and a boundary condition based on a Dirichlet-to-Neumann operator. We work in a fixed domain and a fixed functional space. The variation of the Lagrange operator with respect to the size of the hole $\epsilon$ is given by the variation of the Dirichlet-to-Neumann operator. Calculating this variation requires the knowledge of the expansion of the solution associated to the Dirichlet-to-Neumann problem with respect to $\epsilon$.

The second generalization of the adjoint technique is presented in section 4 . The Lagrange operator does not admit a Frechet-like expansion. In this case, it is necessary to consider its total variation and to take into account the asymptotic expansion of the state. We give at the end of this section some applications of the topological asymptotic expansion to crack detection. The topological gradient at the first iteration gives a good localization of the cracks.

When an iterative algorithm is needed, we refer to [GGM01, GuS01, Mas02, SAM03, HaM04, Ams05] for the presentation of a fixed point method based on the work of Céa et al. [CGM73].

\section{From differential calculus to 0-1 optimization}

We show in this section that it is possible, under weak hypotheses, to derive topological asymptotic expansion from classical gradient.

Let us consider a bounded domain $\Omega \subset \mathbf{R}^{N}(N=1,2,3)$ and the elliptic problem

$$
\left\{\begin{array}{lr}
\nabla \cdot(\alpha \nabla u)+\beta u=b & \text { in } \Omega, \\
B . C . & \text { on } \partial \Omega .
\end{array}\right.
$$


where $\alpha$ and $\beta$ are two functions defined in $\Omega$. The functions $\alpha, \beta$, and the boundary condition B.C. will be specified so that the problem (1) and its topological perturbations admit one and only one solution.

If $\alpha$ goes to 0 in $\omega \subset \subset \Omega$, the corresponding solution $u_{\alpha}$ tends to the solution $u_{N}$ of the problem with Neumann boundary condition:

$$
\left\{\begin{array}{lr}
\nabla \cdot\left(\alpha \nabla u_{N}\right)+\beta u_{N}=b & \text { in } \Omega \backslash \omega, \\
\partial_{n} u_{N}=0 & \text { on } \partial \omega, \\
B . C . & \text { on } \partial \Omega .
\end{array}\right.
$$

If $\beta$ goes to $\infty$ in $\omega \subset \subset \Omega$ the corresponding solution tends to the solution $u_{D}$ of the problem with Dirichlet boundary condition:

$$
\left\{\begin{array}{lr}
\nabla \cdot\left(\alpha \nabla u_{D}\right)+\beta u_{D}=b & \text { in } \Omega \backslash \omega \\
u_{D}=0 & \text { on } \partial \omega, \\
B . C . & \text { on } \partial \Omega .
\end{array}\right.
$$

This second case recalls the standard penalization method used in finite elements methods for the implementation of a Dirichlet condition.

\subsection{From classical gradient to topological sensitivity}

Let $1 \leq p<2$, we denote by $\mathcal{J}$ a differentiable functional on $L^{p}(\Omega)$

$$
\begin{aligned}
\mathcal{J}: L^{p}(\Omega) & \longrightarrow \mathbf{R} \\
c & \longmapsto \mathcal{J}(c)
\end{aligned}
$$

and let $g \in L^{p^{\prime}}(\Omega)$ be the Riesz representation of its differential $\mathcal{J}^{\prime}(c)$. For all $\delta c \in$ $L^{p}(\Omega)$ we have:

$$
\mathcal{J}(c+\delta c)=\mathcal{J}(c)+\int_{\Omega} g(x) \delta c(x) d x+o\left(\|\delta c\|_{p}\right) .
$$

We wish to study the variation of the functional $\mathcal{J}$ with respect to a finite topological perturbation $\delta c_{\epsilon}$. What we have in mind is a perturbation of a finite value in a region of small volume, described as follows: let $\kappa$ be a real

$$
\delta c_{\epsilon}= \begin{cases}\kappa & \text { in } B\left(x_{0}, \epsilon\right) \\ 0 & \text { elsewhere. }\end{cases}
$$

Hypothesis 1 Let $1 \leq p<2$. We make the following assumptions:

1-a) there exists a constant $\gamma_{1}>0$ such that for all $c \in L^{p}(\Omega)$ and for all $\delta c \in$ $L^{p}(\Omega)$ we have:

$$
\left|\mathcal{J}(c+\delta c)-\mathcal{J}(c)-\mathcal{J}^{\prime}(c) . \delta c\right| \leq \gamma_{1}\|\delta c\|_{p}{ }^{2}
$$


1-b) the function $g$ is Lipschitz continuous, in other words there is a constant $\gamma_{2}>0$ such that for all $x, y \in \Omega$ :

$$
|g(x)-g(y)| \leq \gamma_{2}|| x-y|| .
$$

Theorem 1 Let $\delta c_{\epsilon}$ be defined by (2) and the cost function $j$ defined by

$$
j(\epsilon)=\mathcal{J}\left(c+\delta c_{\epsilon}\right) .
$$

If hypothesis I holds, then when $\epsilon \rightarrow 0$,

$$
j(\epsilon)=j(0)+\kappa \rho(\epsilon) g\left(x_{0}\right)+o(\rho(\epsilon)),
$$

where $\rho(\epsilon)=$ meas $\left(B\left(x_{0}, \epsilon\right)\right)$.

The function $g$ is called the topological gradient (of the cost function $\mathcal{J}$, relative to a jump of $\kappa$ ).

Proof: The perturbation $\delta c_{\epsilon}$ given by (2) is small in $L^{p}(\Omega)$ when $\epsilon \rightarrow 0$ since

$$
\left\|\delta c_{\epsilon}\right\|_{p}=\kappa \rho(\epsilon)^{1 / p} .
$$

The right-hand side in hypothesis 1-a) is then

$$
\left\|\delta c_{\epsilon}\right\|_{p}^{2}=\left(\kappa \rho(\epsilon)^{1 / p}\right)^{2}=\kappa^{2} \rho(\epsilon)^{2 / p}=o(\rho(\epsilon)),
$$

since $p<2$. The derivative $\mathcal{J}^{\prime}(c) . \delta c_{\epsilon}$ can be estimated as follows using hypothesis 1-b):

$\left|\mathcal{J}^{\prime}(c) . \delta c_{\epsilon}-\kappa g\left(x_{0}\right) \rho(\epsilon)\right|=\left|\int_{\Omega} g \delta c_{\epsilon}-\kappa g\left(x_{0}\right) \rho(\epsilon)\right| \leq \int_{B\left(x_{0}, \epsilon\right)} \kappa\left|g(x)-g\left(x_{0}\right)\right| d x \leq \gamma_{2} \kappa \epsilon \rho(\epsilon)$.

These two results give:

$$
\left|j(\epsilon)-j(0)-\kappa \rho(\epsilon) g\left(x_{0}\right)\right|=o(\rho(\epsilon)) .
$$

\subsection{The Dirichlet condition}

Let us consider a $C^{1}$ bounded domain $\Omega \subset \mathbf{R}^{N}$, with $N=1,2$ or 3 and let $\mathcal{V} \subset$ $H^{1}(\Omega)$ be a Hilbert space. Let $a$ be a bilinear, continuous and coercive form on $\mathcal{V}$.

For all $c \in L^{p}(\Omega)$, we denote by $a_{c} \in \mathcal{L}_{2}(\mathcal{V})$ the bilinear form defined on $\mathcal{V}$ by:

$$
\begin{aligned}
a_{c}: \mathcal{V} \times \mathcal{V} & \longrightarrow \mathbf{R} \\
(u, v) & \longmapsto a_{c}(u, v)=a(u, v)+\int_{\Omega} c u v d x
\end{aligned}
$$


Lemma 1 The map

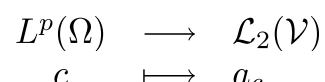

is continuous for

$$
\left\{\begin{array}{cc}
p \geq 1 & \text { when } N=1 \\
p>1 & \text { when } N=2 \\
p>3 / 2 & \text { when } N=3
\end{array}\right.
$$

Since it is an affine map, it is then (twice) differentiable on $L^{p}(\Omega)$.

Proof: It follows from Rellich-Kondrachov's theorem (see e.g. [Bre83]), that $H^{1}(\Omega) \subset$ $L^{q}(\Omega)$ for

$$
\left\{\begin{array}{cc}
1 \leq q \leq \infty & \text { when } N=1 \\
1 \leq q<\infty & \text { when } N=2 \\
1 \leq q<6 & \text { when } N=3
\end{array}\right.
$$

Let $q$ satisfy (5), and let $p$ be such that $\frac{1}{p}+\frac{2}{q}=1$. We have for $u, v \in \mathcal{V}$ :

$$
\begin{aligned}
\left|a_{c}(u, v)-a_{c^{\prime}}(u, v)\right|=\left|\int_{\Omega}\left(c-c^{\prime}\right) u v\right| & \leq\left\|c-c^{\prime}\right\|_{p}\|u v\|_{q / 2} \\
& \leq\left\|c-c^{\prime}\right\|_{p}\|u\|_{q}\|v\|_{q} \\
& \leq K\left\|c-c^{\prime}\right\|\left\|_{p}\right\| u\left\|_{\mathcal{V}}\right\| v \|_{\mathcal{V}}
\end{aligned}
$$

where the two first inequalities follow from Hölder's inequality and the last one from the continuity of the inclusion $\mathcal{V} \subset L^{q}(\Omega)$.

For $N=1,1 \leq q \leq \infty$ gives $p>1$.

For $N=2,1 \leq q<\infty$ gives $p \geq 1$.

For $N=3,1 \leq q<6$ gives $1<p<3 / 2$.

Let $\ell$ be a linear continuous form on $\mathcal{V}$ and $u_{c} \in \mathcal{V}$ be the unique solution of the following variational problem:

$$
a_{c}\left(u_{c}, v\right)=\ell(v) \quad \forall v \in \mathcal{V} .
$$

Lemma 2 Let $p$ satisfy (4) and $J$ be a differentiable functional defined on $\mathcal{V}$, we consider the cost-function

$$
\begin{aligned}
\mathcal{J}: L^{p}(\Omega) & \longrightarrow \mathbf{R} \\
c & \longmapsto \mathcal{J}(c)=J\left(u_{c}\right) .
\end{aligned}
$$

Let $u_{c}$ be the direct state solution of (6), and let $p_{c} \in \mathcal{V}$ be the adjoint state solution of the adjoint problem

$$
a_{c}\left(\psi, p_{c}\right)=-D J\left(u_{c}\right) \cdot \psi \quad \forall \psi \in \mathcal{V}
$$


Then the functional $\mathcal{J}$ is differentiable and for all $\delta c \in L^{p}(\Omega)$ :

$$
\mathcal{J}^{\prime}(c) . \delta c=\int_{\Omega} \delta c \cdot u_{c} \cdot p_{c} d x
$$

In other words, the Riesz representation of the differential g of $\mathcal{J}$ is

$$
g=u_{c} p_{c} .
$$

Proof: We use the Lagrangian method introduced in [Lio76] and [Cea86]. Let us consider the Lagrangian $\mathcal{L}$ defined on $L^{p}(\Omega) \times \mathcal{V} \times \mathcal{V}$ by

$$
\mathcal{L}(c, u, v)=J(u)+a_{c}(u, v)-\ell(v) .
$$

The Lagrangian $\mathcal{L}$ admits partial differentials with respect to each of the 3 variables and we have:

$$
D_{1} \mathcal{L}(c, u, v) \cdot \delta c=a_{\delta c}(u, v)-a(u, v), \quad D_{2} \mathcal{L}(c, u, v) \cdot \phi=D J(u) \cdot \phi+a_{c}(\phi, v) .
$$

Moreover, the map $L^{p} \rightarrow \mathcal{V}, c \mapsto u_{c}$ is differentiable because of lemma 1, equation (6) and implicit functions theorem. When $v \in \mathcal{V}$ is fixed, for every $c \in L^{p}(\Omega)$ we have $\mathcal{J}(c)=J\left(u_{c}\right)=\mathcal{L}\left(c, u_{c}, v\right)$. It follows that for every $\delta c \in L^{p}(\Omega)$,

$$
\mathcal{J}^{\prime}(c) . \delta c=D_{1} \mathcal{L}\left(c, u_{c}, v\right) \cdot \delta c+D_{2} \mathcal{L}\left(c, u_{c}, v\right) . D_{c}\left(u_{c}\right) . \delta c .
$$

Note that if $v=p_{c}$ then $D_{2} \mathcal{L}\left(c, u_{c}, p_{c}\right)=0$, hence

$$
\mathcal{J}^{\prime}(c) . \delta c=D_{1} \mathcal{L}\left(c, u_{c}, p_{c}\right)=\int_{\Omega} \delta c \cdot u_{c} \cdot p_{c} d x .
$$

Theorem 2 Let $p<2$ satisfy (4). Let $a_{c}$ be given by (3) and $u_{c}$ the solution of (6). We assume that $J$ is twice differentiable on $\mathcal{V}$ and consider the particular case of $c \equiv 0$ and $\delta c=\delta c_{\epsilon}$ defined by (2). We assume that the direct state $u_{0}$ and the adjoint state $p_{0}$ are such that $u_{0} p_{0}$ is a Lipschitzian function.

The cost function $j$ defined by $j(\epsilon)=\mathcal{J}\left(u_{\delta c_{\epsilon}}\right)$ has the following expansion:

$$
j(\epsilon)-j(0)=\kappa \rho(\epsilon) u_{0} \cdot p_{0}+o(\rho(\epsilon)) .
$$

Proof : Lemma 2 proves that $\mathcal{J}$ is differentiable on $\mathcal{V}$. When looking at the proof, it appears that it is even twice differentiable, hence hypothesis 1-a) holds, and $\mathcal{J}^{\prime}(0)=u_{0} p_{0}$. Our assumptions imply that hypothesis 1-b) is also satisfied. The asymptotic expansion of $j$ follows from theorem 1 .

When the parameter $\kappa$ is large, the solution $u_{\delta c_{\epsilon}}$ is close to zero in $B\left(x_{0}, \epsilon\right)$. A penalization technique provides a good approximation of the solution in $\Omega \backslash B\left(x_{0}, \epsilon\right)$ with a 
homogeneous Dirichlet condition on the boundary of the hole $B\left(x_{0}, \epsilon\right)$. The topological gradient $g=u_{0} p_{0}$ is exactly what is obtained in next section using a more sophisticated approach (see also [GGM01, GuS01, HaM04, Mas02, SAM03]).

We consider now two one-dimensional examples, where the solution can be explicitly calculated. In the first example the classical gradient is equal to the topological gradient. In the second example, the classical gradient and the topological gradient are different.

\subsection{First example}

Consider the following one-dimensional elliptic state equation:

$$
\left\{\begin{array}{l}
-u^{\prime \prime}+c u=0 \quad \text { for } 0<x<1 \\
u(0)=0 \\
u^{\prime}(1)=1
\end{array}\right.
$$

its variational formulation is

$$
\left\{\begin{array}{l}
\text { find } u \in \mathcal{V} \text { such that } \\
\int_{0}^{1} u^{\prime}(x) w^{\prime}(x) d x+\int_{0}^{1} c(x) u(x) w(x) d x=w(1) \quad \forall w \in \mathcal{V},
\end{array}\right.
$$

where $\mathcal{V}=\left\{w \in H^{1}(0,1) \mid w(0)=0\right\}$.

Let $u_{c} \in \mathcal{V}$ be the unique solution of the problem (9). We want to compute the sensitivity of the functional

$$
\begin{aligned}
\mathcal{J}: L^{1}(0,1) & \longrightarrow \mathbf{R} \\
c & \longmapsto u_{c}(1) .
\end{aligned}
$$

The associated Lagrangian is

$$
\mathcal{L}(c, u, w)=u(1)+\int_{0}^{1} u^{\prime}(x) w^{\prime}(x) d x+\int_{0}^{1} c(x) u(x) w(x) d x-w(1) .
$$

The adjoint state is $p_{c}=-u_{c}$. It follows from lemma 2 that for all $\delta c \in L^{1}(0,1)$,

$$
\mathcal{J}^{\prime}(c) . \delta c=-\int_{0}^{1} \delta c(x) u_{c}^{2}(x) d x
$$

Let us detail this result with $c \equiv 0$ and $\delta c_{\epsilon}$ defined by:

$$
\delta c_{\epsilon}=\left\{\begin{array}{rr}
1 & \text { if } x \in\left[x_{0}, x_{0}+\epsilon\right] \\
0 & \text { elsewhere }
\end{array}\right.
$$


The direct state $u_{0}$ is defined by $u_{0}(x)=x$ for all $x \in[0,1]$. According to theorem 2 , the variation of the cost function $j$ defined by $j(\epsilon)=\mathcal{J}\left(\delta c_{\epsilon}\right)$ is:

$$
j(\epsilon)-j(0)=-\epsilon x_{0}^{2}+o(\epsilon) .
$$

Let us check this result. When $c=\delta c_{\epsilon}$ the solution $u_{\epsilon}=u_{\delta c_{\epsilon}}$ can be explicitly computed:

$$
u_{\epsilon}(x)=\left\{\begin{array}{lr}
\frac{2 x}{\left(x_{0}+1\right) \exp (\epsilon)-\left(x_{0}-1\right) \exp (-\epsilon)} & \text { if } x \in\left[0, x_{0}\right] \\
\frac{\left(x_{0}+1\right) \exp \left(x-x_{0}\right)+\left(x_{0}-1\right) \exp \left(x_{0}-x\right)}{\left(x_{0}+1\right) \exp (\epsilon)-\left(x_{0}-1\right) \exp (-\epsilon)} & \text { if } x \in\left[x_{0}, x_{0}+\epsilon\right] \\
x-\left(x_{0}+\epsilon\right)+\frac{\left(x_{0}+1\right) \exp (\epsilon)+\left(x_{0}-1\right) \exp (-\epsilon)}{\left(x_{0}+1\right) \exp (\epsilon)-\left(x_{0}-1\right) \exp (-\epsilon)} & \text { if } x \in\left[x_{0}+\epsilon, 1\right]
\end{array}\right.
$$

Therefore

$$
\begin{aligned}
j(\epsilon)-j(0) & =u_{\epsilon}(1)-u_{0}(1) \\
& =-x_{0}^{2} \epsilon+x_{0}\left(x_{0}^{2}-1\right) \epsilon^{2}+o\left(\epsilon^{2}\right)
\end{aligned}
$$

This confirms the result obtained using theorem 2 .

\subsection{Example 2}

Let us consider the problem

$$
\left\{\begin{array}{l}
\left.\left(c u^{\prime}\right)^{\prime}=0 \quad \text { in }\right] 0,1[ \\
u(0)=0 \\
c(1) u^{\prime}(1)=1,
\end{array}\right.
$$

for $c \in L^{\infty}(0,1)$, and the cost function $\mathcal{J}(c)=u_{c}(1)$, where $u_{c}$ is the solution to (10).

It is straightforward to check that the adjoint state is $p_{c}=-u_{c}$, and that

$$
\mathcal{J}^{\prime}(c) \delta c=-\int_{0}^{1} \delta c(x)\left(u_{c}^{\prime}(x)\right)^{2} d x .
$$

We consider $c \equiv c_{0}$ (a constant) and the perturbation defined by

$$
\delta c_{\epsilon}=\left\{\begin{array}{rr}
\kappa & \text { if } x \in\left[x_{0}, x_{0}+\epsilon\right] \\
0 & \text { elsewhere. }
\end{array}\right.
$$


After calculation of the exact solution $u_{\epsilon}$, the value of the cost function is found to be

$$
u_{\epsilon}(1)=\frac{1-\epsilon}{c_{0}}+\frac{\epsilon}{c_{0}+\kappa}
$$

hence

$$
j(\epsilon)-j(0)=\epsilon\left(\frac{1}{c_{0}+\kappa}-\frac{1}{c_{0}}\right)
$$

On the other hand, the value of the gradient $\mathcal{J}^{\prime}(c) . \delta c$ is

$$
-\epsilon\left(u_{c}^{\prime}\right)^{2} \kappa=-\epsilon \frac{\kappa}{c_{0}^{2}}
$$

Important remark: We observe that the classical gradient (12) is different from the topological gradient obtained in (11). In this example, theorem 1 can not be applied. The only possibility for the bilinear form $a_{c}$ to be continuous is to have $c \in L^{\infty}$. The requirement in theorem 1 that $c \in L^{p}$ with $1 \leq p<2$ is not met.

Note that if $\kappa$ is small the expressions (11) and (12) are close, but when $\kappa$ is close to $-c_{0}$ the classical gradient (12) remains bounded, while the topological gradient (11) goes to infinity .

\section{First generalized adjoint method}

In this section, we present a more general framework for topological sensitivity than theorem 2, since the conditions of application of this theorem appear to be restrictive in many interesting cases. In section 2.4 , we noted that when the parameters belong to $L^{\infty}$, the classical gradient is inadequate. We consider here the solution of a variational problem in a fixed Hilbert space, where both the bilinear and the linear form depend on a parameter. We give in theorem 3 the variation of a differentiable cost function. This section ends with an example and a counterexample.

\subsection{The general frame}

Let $\mathcal{V}$ be a fixed Hilbert space, and $\mathcal{L}(\mathcal{V})$ denote the space of linear continuous forms on $\mathcal{V}$ and $\mathcal{L}_{2}(\mathcal{V})$ the space of bilinear continuous forms on $\mathcal{V}$. For $\epsilon \geq 0$, let $a_{\epsilon} \in \mathcal{L}_{2}(\mathcal{V})$ and $\ell_{\epsilon} \in \mathcal{L}(\mathcal{V})$.

Hypothesis 2 We make the following assumptions: 
2-a) There exists a real function $f$, a bilinear form $\delta_{a} \in \mathcal{L}_{2}(\mathcal{V})$ and a linear form $\delta_{\ell} \in \mathcal{L}(\mathcal{V})$ such that

$$
\begin{array}{r}
f(\epsilon) \longrightarrow 0 \quad \text { when } \epsilon \rightrightarrows 0 \\
\left\|a_{\epsilon}-a_{0}-f(\epsilon) \delta_{a}\right\|_{\mathcal{L}_{2}(\mathcal{V})}=o(f(\epsilon)) \\
\left\|\ell_{\epsilon}-\ell_{0}-f(\epsilon) \delta_{\ell}\right\|_{\mathcal{L}(\mathcal{V})}=o(f(\epsilon))
\end{array}
$$

2-b) the bilinear form $a_{0}$ is coercive : there exists a constant $\alpha>0$ such that

$$
\forall u \in \mathcal{V}, \quad a_{0}(u, u) \geq \alpha\|u\|^{2} .
$$

According to (14), the bilinear form $a_{\epsilon}$ depends continuously on $\epsilon$, hence there exist $\epsilon_{0}>0$ and $\beta>0$ such that for every $\epsilon \in\left[0, \epsilon_{0}\right]$, the following uniform ellipticity condition holds:

$$
\forall u \in \mathcal{V}, \quad a_{\epsilon}(u, u) \geq \beta\|u\|^{2} .
$$

According to Lax-Milgram's theorem, for $\epsilon \in\left[0, \epsilon_{0}\right]$ the following problem has a unique solution :

$$
\left\{\begin{array}{l}
\text { find } u_{\epsilon} \in \mathcal{V} \text { such that } \\
a_{\epsilon}\left(u_{\epsilon}, v\right)=\ell_{\epsilon}(v), \quad \forall v \in \mathcal{V} .
\end{array}\right.
$$

Lemma 3 If hypothesis 2 holds, then

$$
\left\|u_{\epsilon}-u_{0}\right\|_{\mathcal{V}}=O(f(\epsilon)) .
$$

\subsection{A generalized adjoint method}

We consider now a cost function $j(\epsilon)=J\left(u_{\epsilon}\right)$, where the functional $J$ is differentiable: for every $u \in \mathcal{V}$ there exists a linear continuous form $D J(u) \in \mathcal{L}(\mathcal{V})$ such that

$$
J(u+h)=J(u)+D J(u) \cdot h+o\left(\|h\|_{\mathcal{V}}\right) .
$$

Under hypothesis 2-b), the adjoint problem:

$$
\left\{\begin{array}{l}
\text { find } p \in \mathcal{V} \text { such that } \\
a_{0}(\psi, p)=-D J\left(u_{0}\right)(\psi), \quad \forall \psi \in \mathcal{V} .
\end{array}\right.
$$

admits a unique solution $p_{0}$ called the adjoint state.

For $\epsilon \geq 0$ we define the Lagrangian operator $\mathcal{L}_{\epsilon}$ by

$$
\mathcal{L}_{\epsilon}(u, v)=J(u)+a_{\epsilon}(u, v)-\ell_{\epsilon}(v), \quad \text { for } u, v \in \mathcal{V} .
$$

The asymptotic expansion of $j: j(\epsilon)=J\left(u_{\epsilon}\right)$ is given by the following 
Theorem 3 If hypothesis 2 holds then

$$
j(\epsilon)-j(0)=f(\epsilon) \delta_{\mathcal{L}}\left(u_{0}, p_{0}\right)+o(f(\epsilon)),
$$

where $u_{0}$ is the solution of (16) for $\epsilon=0, p_{0}$ is the solution of (18), and

$$
\forall u, v \in \mathcal{V}, \quad \delta_{\mathcal{L}}(u, v)=\delta_{a}(u, v)-\delta_{\ell}(v) .
$$

Proof: For all $v \in \mathcal{V}$ and $\epsilon \geq 0$,

$$
j(\epsilon)=\mathcal{L}_{\epsilon}\left(u_{\epsilon}, v\right) .
$$

Setting $v=p_{0}$ we obtain

$$
\begin{aligned}
j(\epsilon)-j(0)= & \mathcal{L}_{\epsilon}\left(u_{\epsilon}, p_{0}\right)-\mathcal{L}_{0}\left(u_{0}, p_{0}\right) \\
= & J\left(u_{\epsilon}\right)-J\left(u_{0}\right)+a_{\epsilon}\left(u_{\epsilon}, p_{0}\right)-a_{0}\left(u_{0}, p_{0}\right)+\ell_{0}\left(p_{0}\right)-\ell_{\epsilon}\left(p_{0}\right) \\
= & J\left(u_{\epsilon}\right)-J\left(u_{0}\right)+\left[a_{\epsilon}\left(u_{\epsilon}, p_{0}\right)-a_{0}\left(u_{\epsilon}, p_{0}\right)+a_{0}\left(u_{\epsilon}-u_{0}, p_{0}\right)\right] \\
& -\left[\ell_{\epsilon}\left(p_{0}\right)-\ell_{0}\left(p_{0}\right)-f(\epsilon) \delta_{\ell}\left(p_{0}\right)\right]-f(\epsilon) \delta_{\ell}\left(p_{0}\right) .
\end{aligned}
$$

Using (17), we know that

$$
J\left(u_{\epsilon}\right)-J\left(u_{0}\right)=D J\left(u_{0}\right) \cdot\left(u_{\epsilon}-u_{0}\right)+o\left(\left\|u_{\epsilon}-u_{0}\right\|_{\mathcal{V}}\right) .
$$

It follows that

$$
\begin{aligned}
j(\epsilon)-j(0)= & a_{\epsilon}\left(u_{\epsilon}, p_{0}\right)-a_{0}\left(u_{\epsilon}, p_{0}\right)+a_{0}\left(u_{\epsilon}-u_{0}, p_{0}\right)+D J\left(u_{0}\right) \cdot\left(u_{\epsilon}-u_{0}\right) \\
& +o\left(\left\|u_{\epsilon}-u_{0}\right\|_{\mathcal{V}}\right)-\left[\ell_{\epsilon}\left(p_{0}\right)-\ell_{0}\left(p_{0}\right)-f(\epsilon) \delta_{\ell}\left(p_{0}\right)\right]-f(\epsilon) \delta_{\ell}\left(p_{0}\right) .
\end{aligned}
$$

But $p_{0}$ is the adjoint state, hence

$$
\begin{aligned}
j(\epsilon)-j(0)= & a_{\epsilon}\left(u_{\epsilon}, p_{0}\right)-a_{0}\left(u_{\epsilon}, p_{0}\right)+o\left(\left\|u_{\epsilon}-u_{0}\right\|_{\mathcal{V}}\right) \\
& -\left[\ell_{\epsilon}\left(p_{0}\right)-\ell_{0}\left(p_{0}\right)-f(\epsilon) \delta_{\ell}\left(p_{0}\right)\right]-f(\epsilon) \delta_{\ell}\left(p_{0}\right) . \\
= & \left(a_{\epsilon}-a_{0}\right)\left(u_{0}, p_{0}\right)+\left(a_{\epsilon}-a_{0}\right)\left(u_{\epsilon}-u_{0}, p_{0}\right)+o\left(\left\|u_{\epsilon}-u_{0}\right\|_{\mathcal{V}}\right) \\
& -\left[\ell_{\epsilon}\left(p_{0}\right)-\ell_{0}\left(p_{0}\right)-f(\epsilon) \delta_{\ell}\left(p_{0}\right)\right]-f(\epsilon) \delta_{\ell}\left(p_{0}\right) .
\end{aligned}
$$

It follows from hypothesis 2-a) that

$$
\begin{aligned}
j(\epsilon)-j(0)= & f(\epsilon) \delta_{a}\left(u_{0}, p_{0}\right)+o(f(\epsilon))+f(\epsilon) \delta_{a}\left(u_{\epsilon}-u_{0}, p_{0}\right)+o(f(\epsilon))\left\|u_{\epsilon}-u_{0}\right\|_{\mathcal{V}} \\
& +o\left(\left\|u_{\epsilon}-u_{0}\right\|_{\mathcal{V}}\right)-f(\epsilon) \delta_{\ell}\left(p_{0}\right) .
\end{aligned}
$$


Finally, it follows from lemma 3, the continuity of $\delta_{a}$ and the fact that $f(\epsilon) \rightarrow 0$ when $\epsilon \rightarrow 0$ that

$$
j(\epsilon)=j(0)+f(\epsilon)\left[\delta_{a}\left(u_{0}, p_{0}\right)-\delta_{\ell}\left(p_{0}\right)\right]+o(f(\epsilon)) .
$$

The generalized Lagrangian technique presented above have been applied to the several problems, we refer the reader to [GGM01, GuS01, Mas02, SAM03, HaM04, MPS05]. To satisfy the assumptions of hypothesis 2 , we consider a domain truncation technique that we present in the next section on an example.

\subsection{Example 3: Dirichlet condition for Poisson equation}

We expose here the calculation of the topological sensitivity for a hole with a Dirichlet condition for the Poisson equation. We describe the domain truncation technique [GGM01, GuS01, Mas02, SAM03, HaM04, MPS05] that allows to work in a fixed Hilbert space and to satisfy Hypothesis 2 .

Let $\Omega \subset \mathbf{R}^{2}$ with $C^{1}$ boundary. The boundary of $\Omega$ is decomposed in two parts $\partial \Omega=$ $\Gamma_{D} \cup \Gamma_{N}$, with strictly positive measure. We consider a source term $h \in H_{00}^{1 / 2}\left(\Gamma_{N}\right)^{\prime}$. Let $u_{\Omega} \in H^{1}(\Omega)$ be the unique solution of

$$
\left\{\begin{array}{lr}
\Delta u=0 & \text { in } \Omega \\
u=0 & \text { on } \Gamma_{D} \\
\partial_{n} u=h & \text { on } \Gamma_{N},
\end{array}\right.
$$

where $\partial_{n} u$ is the normal derivative of $u$. Let $J$ be a differentiable function on $H^{1}(\Omega)$.

Our aim is to derive the topological sensitivity of $J$ relatively to a circular perforation of radius $\epsilon$ with Dirichlet condition at some point $x \in \Omega$. The point $x \in \Omega$ is fixed, $\Omega_{\epsilon}=\Omega \backslash B(x, \epsilon)$ is the perforated domain (for $\epsilon$ small enough). The solution in the perforated domain is given by the problem

$$
\left\{\begin{array}{lr}
\Delta u_{\Omega_{\epsilon}}=0 & \text { in } \Omega_{\epsilon} \\
u_{\Omega_{\epsilon}}=0 & \text { on } \Gamma_{D} \\
u_{\Omega_{\epsilon}}=0 & \text { on } \Sigma_{\epsilon} \\
\partial_{n} u_{\Omega_{\epsilon}}=h & \text { on } \Gamma_{N},
\end{array}\right.
$$

where $\Sigma_{\epsilon}$ is the boundary of $B(x, \epsilon)$.

Let $R>\epsilon$ be such that $B(x, R) \subset \Omega$. The boundary of $B(x, R)$ is denoted $\Sigma_{R}$ and $D_{\epsilon}$ denotes the corona $B(x, R) \backslash B(x, \epsilon)$, see figure 1 .

For a given $\psi \in H^{1 / 2}\left(\Sigma_{R}\right)$, we consider $u_{\psi}^{\epsilon}$ the (unique) solution to the problem

$$
\begin{cases}\Delta u_{\psi}^{\epsilon}=0 & \text { in } D_{\epsilon} \\ u_{\psi}^{\epsilon}=\psi & \text { on } \Sigma_{R} \\ u_{\psi}^{\epsilon}=0 & \text { on } \Sigma_{\epsilon},\end{cases}
$$




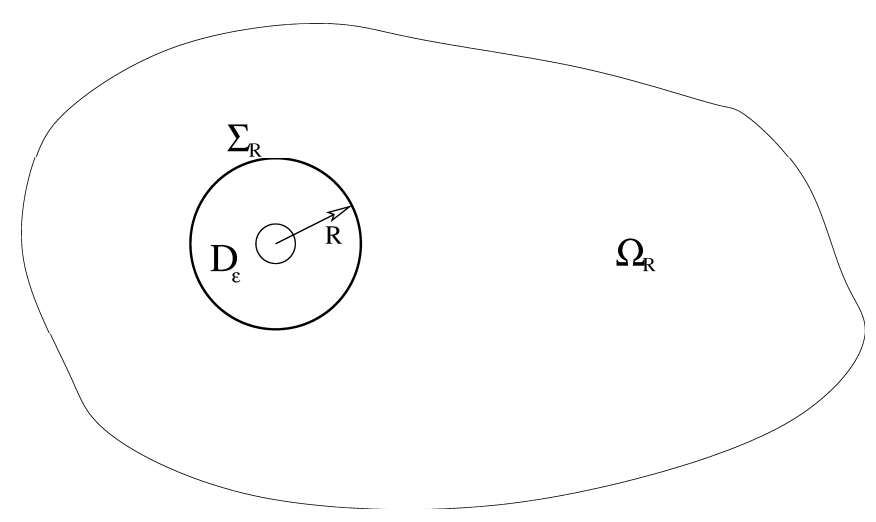

Figure 1. Domain truncation.

and the Dirichlet-to-Neumann operator

$$
\begin{aligned}
T^{\epsilon}: H^{1 / 2}\left(\Sigma_{R}\right) & \longrightarrow H^{-1 / 2}\left(\Sigma_{R}\right) \\
\psi & \longmapsto T^{\epsilon} \psi=\left.\nabla u_{\psi}^{\epsilon} \cdot n\right|_{\Sigma_{R}},
\end{aligned}
$$

where $\left.n\right|_{\Sigma_{R}}$ is the outward normal to the boundary $\Sigma_{R}$.

We consider the truncated problem

$$
\left\{\begin{array}{lc}
\Delta u_{\epsilon}=0 & \text { in } \Omega_{R} \\
u_{\epsilon}=0 & \text { on } \Gamma_{D} \\
\partial_{n} u_{\epsilon}+T^{\epsilon} u_{\epsilon}=0 & \text { on } \Sigma_{R} \\
\partial_{n} u_{\epsilon}=h & \text { on } \Gamma_{N},
\end{array}\right.
$$

the variational formulation associated to $(21)$ is:

$$
\left\{\begin{array}{l}
\text { find } u_{\epsilon} \in \mathcal{V}_{R} \text { such that } \\
a_{\epsilon}\left(u_{\epsilon}, v\right)=\ell(v) \quad \forall v \in \mathcal{V}_{R},
\end{array}\right.
$$

with $\mathcal{V}_{R}=\left\{u \in H^{1}\left(\Omega_{R}\right) \mid u=0\right.$ on $\left.\Gamma_{D}\right\}$ and

$$
a_{\epsilon}(u, v)=\int_{\Omega_{R}} \nabla u . \nabla v d x+\int_{\Sigma_{R}}\left(T^{\epsilon} u\right) v d s, \quad \ell(v)=\int_{\Gamma_{N}} h v d s .
$$

It is standard to prove that (21) has a unique solution in $\mathcal{V}_{R}$ that is the restriction to $\Omega_{R}$ of the solution of (20).

We now have a fixed Hilbert space, as required by theorem 3. It suffices to estimate the variation of the bilinear form $a_{\epsilon}$ (since in this example the linear form $\ell$ is independent of $\epsilon$ ). We have [Mas02]:

$$
a_{\epsilon}(u, v)-a_{0}(u, v)=\int_{\Sigma_{R}}\left(\left(T^{\epsilon}-T^{0}\right) u\right) v d s=f(\epsilon) \bar{u}^{\Sigma_{R}} \bar{p}^{\Sigma_{R}}+o(f(\epsilon)),
$$


where $\bar{u}^{\Sigma_{R}}$, resp. $\bar{p}^{\Sigma_{R}}$, is the mean value of $u$, resp. $p$, on $\Sigma_{R}$. Since $u_{0}$ and $p_{0}$ are harmonic,

$$
\delta a\left(u_{0}, p_{0}\right)=u_{\Omega}(x) \cdot p_{\Omega}(x),
$$

where $u_{\Omega}$ is the solution and $p_{\Omega}$ is the adjoint state in $\Omega$ without a hole.

Using Theorem 3, we obtain the final result

$$
j(\epsilon)-j(0)=f(\epsilon) u\left(x_{0}\right) p\left(x_{0}\right),
$$

the topological gradient $g=u . p$ is exactly what we obtained by Theorem 2 using a straightforward way based on a penalization technique.

\subsection{Example 4: interface between two material properties}

Let $\mathcal{V}=\left\{u \in H^{1}(0,1) \mid u(0)=0\right\}$. For $\left.x_{0} \in\right] 0,1[$ and $\epsilon$ sufficiently small we define

$$
a_{\epsilon}(u, v)=\int_{0}^{x_{0}+\epsilon} c_{1} u^{\prime} v^{\prime} d x+\int_{x_{0}+\epsilon}^{1} c_{2} u^{\prime} v^{\prime} d x
$$

where $c_{1}$ and $c_{2}$ are two positive constants. We consider the following variational problem:

$$
\left\{\begin{array}{l}
\text { find } u \in \mathcal{V} \text { such that } \\
a_{\epsilon}(u, v)=\ell(v) \quad \forall v \in \mathcal{V},
\end{array}\right.
$$

where the linear form $\ell$ is given by: $\ell(v)=v(1)$.

We want to determine the variation of the cost function $j(\epsilon)$ defined by

$$
j(\epsilon)=J\left(u_{\epsilon}\right)=u_{\epsilon}(1) .
$$

The solution of (22) is explicitly given by

$$
u_{\epsilon}(x)= \begin{cases}\frac{1}{c_{1}} x & \text { for } x \in\left[0, x_{0}+\epsilon\right] \\ \frac{1}{c_{1}}\left(x_{0}+\epsilon\right)+\frac{1}{c_{2}}\left(x-x_{0}-\epsilon\right) & \text { for } x \geq x_{0}+\epsilon\end{cases}
$$

It is straightforward to compute $j^{\prime}(\epsilon)$ and check that $j^{\prime}(0)=\frac{1}{c_{1}}-\frac{1}{c_{2}}$. On the other hand, let us compute the variation of the Lagrangian, that is $\delta_{a}$ (since $\delta_{\ell}=0$ ). For $u, v \in \mathcal{V}$,

$$
a_{\epsilon}(u, v)-a_{0}(u, v)=\int_{x_{0}}^{x_{0}+\epsilon}\left(c_{1}-c_{2}\right) u^{\prime} v^{\prime}
$$

When $\epsilon \rightarrow 0$, this quantity is equivalent to $\epsilon\left(c_{1}-c_{2}\right) u^{\prime}\left(x_{0}^{+}\right) v^{\prime}\left(x_{0}^{+}\right)$. Hence $a_{\epsilon}(u, v)-$ $a_{0}(u, v)-\epsilon \delta_{a}(u, v)=o(\epsilon)$ with $\delta_{a}(u, v)=\left(c_{1}-c_{2}\right) u^{\prime}\left(x_{0}^{+}\right) v^{\prime}\left(x_{0}^{+}\right)$. 
Since $D J\left(u_{c}\right) \cdot u=-\ell(u)$, the adjoint state is $p_{0}=-u_{0}$, hence $\delta_{\mathcal{L}}\left(u_{0}, p_{0}\right)=$ $\delta_{a}\left(u_{0}, p_{0}\right)=\left(c_{1}-c_{2}\right) u_{0}^{\prime}\left(x_{0}^{+}\right) v_{0}^{\prime}\left(x_{0}^{+}\right)=\frac{c_{2}-c_{1}}{c_{2}^{2}}$.

Important remark: We have $\delta_{\mathcal{L}}\left(u_{0}, p_{0}\right) \neq j^{\prime}(\epsilon)$. In this example hypothesis 2-a) is not satisfied: the bilinear form $\delta_{a}$ is not continuous, since pointwise evaluation is not continuous on $L^{2}(0,1)$. Theorem 3 can not be applied. We suggest to the reader to apply theorem 3 when a domain truncation method is considered.

\section{Second generalization of the adjoint approach}

As shown in the previous example, in some cases of interest hypothesis 2 does not hold: the variation of the Lagrange operator with respect to the state is not small. An adaptation of theorem 3 is required in order to calculate topological sensitivity. The variation of the cost function is splitted in several terms, namely when the Lagrange operator is defined by

$$
\mathcal{L}(\epsilon, u, v)=J(u)+a_{\epsilon}(u, v)-\ell_{\epsilon}(v)
$$

the variation of cost function is given for any $v \in \mathcal{V}$ by

$$
j(\epsilon)-j(0)=\mathcal{L}\left(\epsilon, u_{\epsilon}, v\right)-\mathcal{L}\left(0, u_{0}, v\right) .
$$

The variation of $j$ must take into account $\partial \mathcal{L} / \partial \epsilon$, but also the variation induced by $u_{\epsilon}-u_{0}$. The variation of $u_{\epsilon}-u_{0}$ has been studied in [AVV01, AVV03, AmK04, MNP00].

See $\lceil$ Ams05] for the nonlinear case.

\subsection{A general framework}

We propose here a general result [ADS04], taking into account the variation of the Lagrange operator via the bilinear form $a_{\epsilon}$, the linear form $\ell_{\epsilon}$, the state $u_{\epsilon}$ and also the cost function $J_{\epsilon}$, in case it depends on $\epsilon$.

Let $\mathcal{V}$ be a Hilbert space and, for $\epsilon \geq 0$, let $a_{\epsilon}$ be a bilinear continuous and coercive form on $\mathcal{V}$ and $\ell_{\epsilon}$ be a linear continuous form on $\mathcal{V}$. Let $u_{\epsilon}$ be the direct state, solution to

$$
\left\{\begin{array}{l}
\text { find } u_{\epsilon} \in \mathcal{V} \text { such that } \\
a_{\epsilon}\left(u_{\epsilon}, v\right)=\ell_{\epsilon}(v), \quad \forall v \in \mathcal{V} .
\end{array}\right.
$$

We consider also a cost function $j(\epsilon)=J_{\epsilon}\left(u_{\epsilon}\right)$, where the functional $J_{\epsilon}$ is differentiable at the point $u_{0}$ : there exists $L_{\epsilon} \in \mathcal{L}(\mathcal{V}, \mathbf{R})$ such that

$$
J_{\epsilon}\left(u_{0}+h\right)=J_{\epsilon}\left(u_{0}\right)+L_{\epsilon}(h)+o\left(\|h\|_{\mathcal{V}}\right) .
$$


Let $p_{\epsilon}$ be the adjoint state, solution to

$$
\left\{\begin{array}{l}
\text { find } p_{\epsilon} \in \mathcal{V} \text { such that } \\
a_{\epsilon}\left(\psi, p_{\epsilon}\right)=-L_{\epsilon}\left(u_{0}\right) \cdot \psi, \quad \forall \psi \in \mathcal{V} .
\end{array}\right.
$$

Hypothesis 3 There exists a real function $f^{\prime}$ tending to zero with $\epsilon$, and four real numbers $\delta_{a}, \delta_{\ell}, \delta J_{1}, \delta J_{2} \in \mathbf{R}$ such that

$$
\begin{aligned}
& \text { 3-a) }\left(a_{\epsilon}-a_{0}\right)\left(u_{0}, p_{\epsilon}\right)=f(\epsilon) \delta_{a}+o(f(\epsilon)), \\
& \text { 3-b) }\left(\ell_{\epsilon}-\ell_{0}\right)\left(p_{\epsilon}\right)=f(\epsilon) \delta_{\ell}+o(f(\epsilon)), \\
& \text { 3-c) } J_{\epsilon}\left(u_{\epsilon}\right)=J_{\epsilon}\left(u_{0}\right)+L_{\epsilon}\left(u_{\epsilon}-u_{0}\right)+f(\epsilon) \delta J_{1}+o(f(\epsilon)), \\
& \text { 3-d) } J_{\epsilon}\left(u_{0}\right)=J_{0}\left(u_{0}\right)+f(\epsilon) \delta J_{2}+o(f(\epsilon)) .
\end{aligned}
$$

The quantity $\delta J_{1}$ takes into account the variation of $u_{\epsilon}-u_{0}$ when it is not $O(f(\epsilon))$, and the quantity $\delta J_{2}$ takes into account the variation of $J_{\epsilon}$ with respect to $\epsilon$.

The topological sensitivity analysis of $j$ is then given by

Theorem 4 If hypothesis 3 holds, then

$$
j(\epsilon)-j(0)=f(\epsilon) \delta j+o(f(\epsilon)),
$$

where $\delta j=\delta J_{1}+\delta J_{2}+\delta a-\delta \ell$.

Proof: We have

$$
j(\epsilon)-j(0)=\left[J_{\epsilon}\left(u_{\epsilon}\right)-J_{0}\left(u_{0}\right)\right]+\left[a_{\epsilon}\left(u_{\epsilon}, p_{\epsilon}\right)-a_{0}\left(u_{0}, p_{\epsilon}\right)\right]-\left[\ell_{\epsilon}(\epsilon)-\ell_{0}\left(p_{\epsilon}\right)\right] .
$$

it follows from hypothesis 3 and the definition of adjoint state that

$$
\begin{aligned}
j(\epsilon)-j(0)= & J_{\epsilon}\left(u_{\epsilon}\right)-J_{0}\left(u_{0}\right)+a_{\epsilon}\left(u_{\epsilon}-u_{0}, p_{\epsilon}\right)+f(\epsilon)\left(\delta a-\delta_{\ell}\right)+o(f(\epsilon)) \\
= & {\left[J_{\epsilon}\left(u_{\epsilon}\right)-J_{\epsilon}\left(u_{0}\right)\right]+\left[J_{\epsilon}\left(u_{0}\right)-J_{0}\left(u_{0}\right)\right]+a_{\epsilon}\left(u_{\epsilon}-u_{0}, p_{\epsilon}\right) } \\
& +f(\epsilon)\left(\delta a-\delta_{\ell}\right)+o(f(\epsilon)) \\
= & a_{\epsilon}\left(u_{\epsilon}-u_{0}, p_{\epsilon}\right)+L_{\epsilon}\left(u_{\epsilon}-u_{0}\right)+f(\epsilon)\left(\delta J_{1}+\delta J_{1}+\delta a-\delta_{\ell}\right)+o(f(\epsilon)) \\
= & f(\epsilon)\left(\delta J_{1}+\delta J_{1}+\delta a-\delta_{\ell}\right)+o(f(\epsilon)) .
\end{aligned}
$$

The topological expansion (Hypothesis 3 ) of $a, \ell$, and $J$ requires the knowledge of the topological asymtotic expansion of $u_{\epsilon}$ and $p_{\epsilon}$. This subject is covered by a huge litterature [KoV87, FrV89, MNP00, Ili92, AVV01, AVV03, AmK04]. In the next section we present an example of application of these methods. 


\subsection{Insertion of inhomogeneities for Poisson equation}

We present in this section the results obtained in [Ams03, ADS04].

\subsubsection{The problem}

We consider $\Omega$ a smooth domain in $\mathbf{R}^{N}, N=2$ or 3 . The boundary of $\Omega$ is divided in two parts of positive measure $\partial \Omega=\Gamma_{N} \cup \Gamma_{D}$. We assume that $\Omega$ contains a small inhomogeneity around the origin $\omega_{\epsilon}=\epsilon B$, where $B \subset \mathbf{R}^{N}$ is a bounded smooth domain containing the origin. We consider the piecewise constant function:

$$
\alpha_{\epsilon}(x)=\left\{\begin{array}{rr}
\alpha_{0} & \text { in } \Omega \backslash \omega_{\epsilon} \\
\alpha_{1} & \text { in } \omega_{\epsilon}
\end{array}\right.
$$

where $\alpha_{0}$ and $\alpha_{1}$ are positive constants. Let $g \in L^{2}(\Gamma)$. For $\epsilon \geq 0$, we denote $u_{\epsilon}$ the solution of the following Poisson problem:

$$
\left\{\begin{array}{lr}
\nabla \cdot\left(\alpha_{\epsilon} \nabla u_{\epsilon}\right)=0 & \text { in } \Omega \\
\alpha_{0} \partial_{n} u_{\epsilon}=g & \text { on } \Gamma_{N}, \\
u_{\epsilon}=0 & \text { on } \Gamma_{D} .
\end{array}\right.
$$

This study is general, since a translation reduces the case of an inhomogeneity located around the point $x_{0}$ to an inhomogeneity located around the origin.

The variational formulation associated to (24) reads

$$
\left\{\begin{array}{l}
\text { find } u_{\epsilon} \in \mathcal{V} \text { such that } \\
a_{\epsilon}\left(u_{\epsilon}, v\right)=\ell(v) \quad \forall v \in \mathcal{V},
\end{array}\right.
$$

where $\mathcal{V}=\left\{u \in H^{1}(\Omega)|u|_{\Gamma_{D}}=0\right\}$ and, for $u, v \in H^{1}(\Omega)$,

$$
a_{\epsilon}(u, v)=\int_{\Omega} \alpha_{\epsilon} \nabla u . \nabla v d x \quad \ell(v)=\alpha_{0} \int_{\partial \Omega} g v d s .
$$

Since $\alpha_{0}$ and $\alpha_{1}$ are positive, the bilinear form $a_{\epsilon}$ is continuous and coercive on $\mathcal{V}$, and $\ell$ is a continuous linear form. Hence problem (25) admits a unique solution.

\subsubsection{Variation of the bilinear form}

Let $\boldsymbol{\Phi}$ be the solution of

$$
\left\{\begin{array}{l}
\Delta \boldsymbol{\Phi}=0 \text { in } B \text { and } \mathbf{R}^{N} \backslash \bar{B} \\
\boldsymbol{\Phi} \text { is continuous across } \partial B \\
\frac{\alpha_{0}}{\alpha_{1}}\left(\partial_{n} \boldsymbol{\Phi}\right)^{+}-\left(\partial_{n} \boldsymbol{\Phi}\right)^{-}=-\mathbf{n} \text { on } \partial \mathrm{B}, \\
\lim _{|y| \rightarrow \infty}|\boldsymbol{\Phi}(y)|=0
\end{array}\right.
$$

where $\mathbf{n}$ denotes the outward unit normal to $\partial B$ and superscript + , resp. - , denotes the limits over $\partial B$ from outside, resp. from inside. 
It is proved in [ADS04] that hypothesis 3-a) is satisfied with $f(\epsilon)=\epsilon^{N}$ and

$$
\delta a=\left(\alpha_{1}-\alpha_{0}\right) \nabla u_{0}(0)^{T}\left[\left(\frac{\alpha_{0}}{\alpha_{1}}-1\right) \int_{\partial B} \mathbf{n} \otimes \mathbf{\Phi}(y) d s(y)+|B| I\right] \nabla p_{0}(0),
$$

where $u \otimes v=u v^{T}$. Let us introduce the matrix

$$
P=\left(\frac{\alpha_{0}}{\alpha_{1}}-1\right) \int_{\partial B} \mathbf{n} \otimes \boldsymbol{\Phi}(y) d s(y)
$$

The matrix $P$ depends only on the shape of $B$ and of the ratio $\alpha_{0} / \alpha_{1}$, and we have

$$
\delta a=\left(\alpha_{1}-\alpha_{0}\right) \nabla u_{0}(0)^{T}(P+|B| I) \nabla v_{0}(0)^{T} .
$$

Unit ball: when $B$ is the unit ball, the matrix $P$ defined in (26) is equal to

$$
P=\frac{\alpha_{0}-\alpha_{1}}{(N-1) \alpha_{0}+\alpha_{1}}|B| I
$$

Ellipse in the plane: when $B$ is the ellipse in the plane with major axis $2 r_{1}$ and minor axis $2 r_{2}$, the matrix $P$ is equal to

$$
P=\pi r_{1} r_{2}\left(\alpha_{0}-\alpha_{1}\right)\left(\begin{array}{cc}
\frac{1}{\alpha_{0} r_{1}+\alpha_{1} r_{2}} & 0 \\
0 & \frac{1}{\alpha_{0} r_{2}+\alpha_{1} r_{1}}
\end{array}\right)
$$

Elliptic hole with homogeneous Neumann condition: it is the limiting case of the ellipse with $\alpha_{1} \rightarrow 0$. We obtain formally a well known result [SoZ99]

$$
P=\pi\left(\begin{array}{cc}
r_{2} & 0 \\
0 & r_{1}
\end{array}\right)
$$

and $\delta a$ is straightforward to estimate.

Straight crack of length $2 r_{1}$ with normal $\mathbf{n}$ : it is formally the limit of the previous case with $r_{2} \rightarrow 0$. After calculations, one obtains

$$
\delta a=-\pi r_{1}\left(\nabla u_{0}(0) \cdot \mathbf{n}\right)\left(\nabla p_{0}(0) \cdot \mathbf{n}\right) .
$$

This formula is proved to be exact using other methods in [AHM05].

The linear form $\ell$ does not depend on $\epsilon$, hence

$$
\delta \ell=0
$$




\subsubsection{Variation of the cost function}

The calculation of $\delta J_{1}$ and $\delta J_{2}$ is given in [ADS04] for different cost functions.

Example 1: the cost function is defined by

$$
J_{\epsilon}(u)=\int_{\Omega} \alpha_{\epsilon}\left|u_{\epsilon}-u_{g}\right|^{2} d x
$$

where $u_{g} \in H^{2}(\Omega)$ is a "target" function.

Hypothesis 3 is satisfied with $f(\epsilon)=\epsilon^{N}, L_{\epsilon}(u)=2 \int_{\Omega} \alpha_{\epsilon} u\left(u_{0}-u_{g}\right) d x$ for all $u \in H^{1}(\Omega), \delta J_{1}=0$ and $\delta J_{2}=\left(\alpha_{1}-\alpha_{0}\right)|B|\left|u_{0}(0)-u_{g}(0)\right|^{2}$.

Example 2: The cost function is defined by

$$
J_{\epsilon}(u)=\int_{\Omega} \alpha_{\epsilon}\left|\nabla\left(u_{\epsilon}-u_{g}\right)\right|^{2} d x
$$

where $u_{g} \in H^{3}(\Omega)$ is a "target" function.

Hypothesis 3 is satisfied with $f(\epsilon)=\epsilon^{N}, L_{\epsilon}(u)=2 \int_{\Omega} \alpha_{\epsilon} \nabla u \nabla\left(u_{0}-u_{g}\right) d x$ for all $u \in H^{1}(\Omega), \delta J_{1}=\left(\alpha_{1}-\alpha_{0}\right) \nabla u_{0}(0)^{T} P \nabla u_{0}(0)$ and $\delta J_{2}=\left(\alpha_{1}-\alpha_{0}\right)|B|\left|\nabla u_{0}(0)-\nabla u_{g}(0)\right|^{2}$, where the matrix $P$ is defined by (26).

\subsection{Crack detection for Poisson equation: numerical results}

We present here results obtained in [AHM05]. A domain contains an unknown crack, and the available data are measures of $u$ and $\partial_{n} u$ on the boundary of the domain, where $u$ satisfies a Poisson equation in the domain and the crack is perfectly insulating.

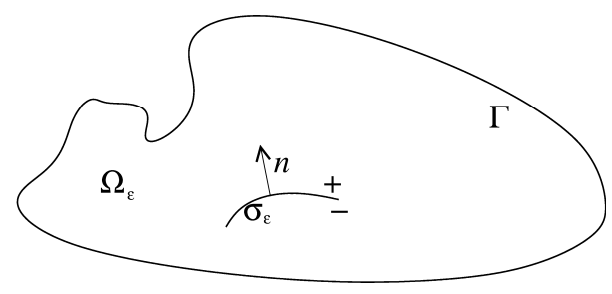

Figure 2. The cracked domain. 


\subsubsection{The problem}

Let $\Omega$ be a domain containing a perfectly insulating crack $\sigma^{*}$ whose location, orientation, shape and length are to be retrieved. We dispose of the temperature $\theta$ measured on the boundary $\Gamma$ for a heat flux $\varphi$ prescribed: $\theta=u\left(\sigma^{*}\right)_{\mid \Gamma}$, where $u\left(\sigma^{*}\right)$ is the solution to

$$
\left\{\begin{array}{rlll}
\Delta u\left(\sigma^{*}\right)= & 0 & \text { in } & \Omega \backslash \overline{\sigma^{*}}, \\
\partial_{n} u\left(\sigma^{*}\right)= & \text { on } & \Gamma, \\
\partial_{n} u\left(\sigma^{*}\right)= & 0 & \text { on } & \sigma^{*} .
\end{array}\right.
$$

To ensure well-posedness of the above system, we assume the normalization condition

$$
\int_{\Gamma} \varphi d s=0
$$

and we impose that the mean value of the solution is equal to zero:

$$
\int_{\Omega \backslash \overline{\sigma^{*}}} u\left(\sigma^{*}\right) d x=0
$$

Since the boundary conditions $(\theta, \varphi)$ are over specified, we can define for any crack $\sigma \subset \Omega$ two direct problems similar to (28): the "Dirichlet" problem with Dirichlet boundary condition $u=\theta$ on $\Gamma$, and the "Neumann" problem with Neumann boundary condition $\partial_{n} u=\varphi$ on $\Gamma$. The solutions of these direct problems are denoted $u_{D}$ and $u_{N}$. The actual crack $\sigma^{*}$ is reached ( $\sigma=\sigma^{*}$ ) when there is no misfit between both solutions, that is, when the cost functional

$$
\mathcal{J}(\sigma)=J\left(u_{D}(\sigma), u_{N}(\sigma)\right)=\frac{1}{2}\left\|u_{D}(\sigma)-u_{N}(\sigma)\right\|_{L^{2}(\Omega)}^{2}
$$

vanishes. This is the so-called Kohn-Vogelius criterion [KoV87]. To compute the corresponding topological gradient, we need to solve numerically:

- two direct problems in the full domain $\Omega$, one Dirichlet problem and one Neumann problem. Their solutions are denoted by $u_{D}$ and $u_{N}$.

- two adjoint problems (defined also in the full domain $\Omega$ ) that are derived directly from their variational formulations. Their solutions are denoted by $p_{D}$ and $p_{N}$.

The following value of the topological sensitivity is a straightforward application of (27), a different proof can be found in [AHM05]:

$$
\mathcal{J}\left(\sigma_{x, \epsilon, \mathbf{n}}\right)-\mathcal{J}(\emptyset)=-\pi \epsilon^{2}\left[\left(\nabla u_{D}(x) \cdot \mathbf{n}\right)\left(\nabla p_{D}(x) \cdot \mathbf{n}\right)+\left(\nabla u_{N}(x) \cdot \mathbf{n}\right)\left(\nabla p_{N}(x) . \mathbf{n}\right)\right]+o\left(\epsilon^{2}\right),
$$

where $\sigma_{x, \epsilon, \mathbf{n}}$ is the line crack of length $2 \epsilon$, centered at the point $x$ and of unit normal $\mathbf{n}$. The corresponding topological gradient

$$
g(x, \mathbf{n})=-\pi\left[\left(\nabla u_{D}(x) \cdot \mathbf{n}\right)\left(\nabla p_{D}(x) \cdot \mathbf{n}\right)+\left(\nabla u_{N}(x) \cdot \mathbf{n}\right)\left(\nabla p_{N}(x) \cdot \mathbf{n}\right)\right]
$$


can be written as follows:

$$
g(x, \mathbf{n})=\mathbf{n}^{T} M(x) \mathbf{n},
$$

where $M(x)$ is the symmetric matrix defined by

$$
\begin{aligned}
M(x)=-\frac{\pi}{2}\left[\nabla u_{D}(x) \nabla p_{D}(x)^{T}+\nabla p_{D}(x) \nabla u_{D}(x)^{T}\right. & +\nabla u_{N}(x) \nabla p_{N}(x)^{T} \\
& \left.+\nabla p_{N}(x) \nabla u_{N}(x)^{T}\right] .
\end{aligned}
$$

According to that expression, $g(x, \mathbf{n})$ is minimal at the point $x$ when the normal $\mathbf{n}=\mathbf{n}_{\mathbf{1}}$ is an eigenvector associated to the smallest eigenvalue $\lambda_{1}(x)$ of the matrix $M(x)$. Then, $g\left(x, \mathbf{n}_{\mathbf{1}}\right)=\lambda_{1}(x)$. We will call topological gradient this value.

\subsubsection{Numerical results in one iteration without noise}
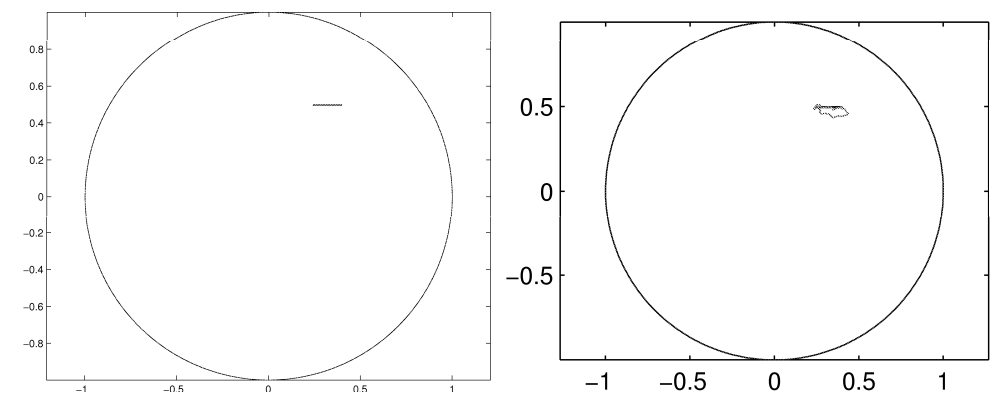

Figure 3. Left : the unknown crack; right : superposition of the actual crack and a negative isovalue of the topological gradient (courtesy S.Amstutz, I.Horchani).

This leads to a simple and very fast numerical procedure. First, the two direct problems and the two adjoint problems (Dirichlet and Neumann) are solved. Then the matrix $M(x)$ and its eigenvalues are computed in each cell of the mesh. By using the previous asymptotic analysis, the crack is expected to lie in the regions where the topological gradient is the most negative.

The heat flux $\varphi$ is imposed on $\Gamma$ by $\varphi(x)=x_{2}$, the second coordinate of the point $x$. In this experiment, the flux inside the full domain is not parallel to the crack, so only one measurement is needed for the reconstruction (see [AnB96]). We apply formula (30), the location of the unknown crack and the topological gradient are indicated in figure 3. We observe that the most negative values of the topological gradient are located near the actual crack. 


\subsubsection{Numerical results in one iteration with noise}

We focus here on simulated noisy measurements. A white noise is added to the exact data. Figure 4 shows the results obtained for $5 \%$, and $20 \%$ of noise. We observe that the inversion procedure is quite robust with respect to the presence of noise in the measurements.

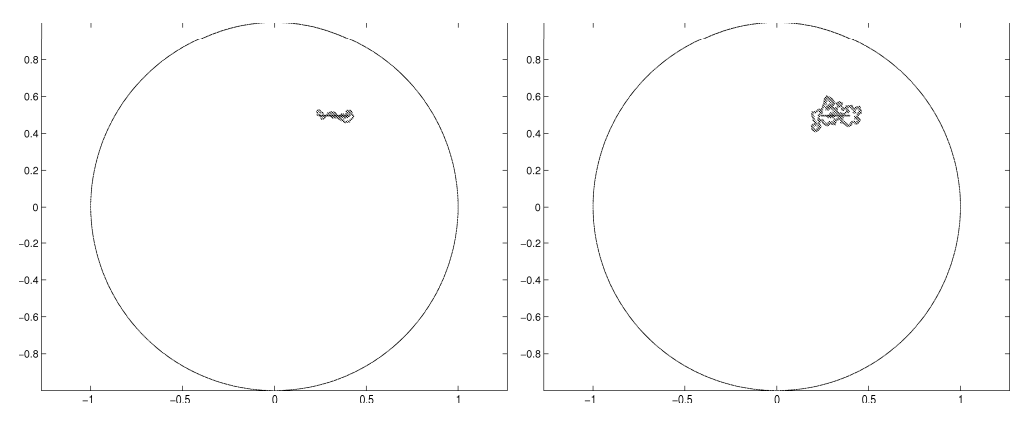

Figure 4. Negative isovalues of the topological gradient. Noise: $5 \%$ (left) and 20\% (right). (courtesy S.Amstutz, I.Horchani).

\subsubsection{Case of multi-cracks}

The computation of the topological gradient does not depend on the number of cracks inside the domain. This remark is illustrated by the following experiment. The actual cracks and the topological gradient are represented in Figure 5. We use now two fluxes $\varphi_{1}(x)=x_{1}$ and $\varphi_{2}(x)=x_{2}$. The cost functional is the sum of the two quadratic misfits. We emphasize again that these results are obtained in only one iteration.

\section{References}

[All02] G. Allaire. Shape optimization by the homogenization method. Springer, Applied Mathematical Sciences Vol. 146, 2002.

[AlK93] G.Allaire and R.Kohn Optimal design for minimum weight and compliance in plane stress using external microstructures, European J.of Mechanics A/SOlids 12(6), 839-878 (1993)

[AlT02] F.Allaire and A.-M. Toader A level-set method for shape optimization, C.R.Acad.Sci. série I (334), 1125-1130, 2002.

[AGJ05] G.Allaire, F.de Gournay, F.Jouve and A.-M. Toader Structural optimization using topological and shape sensitivity via a level set method, to appear in Control and Cybernetics. Inter- 


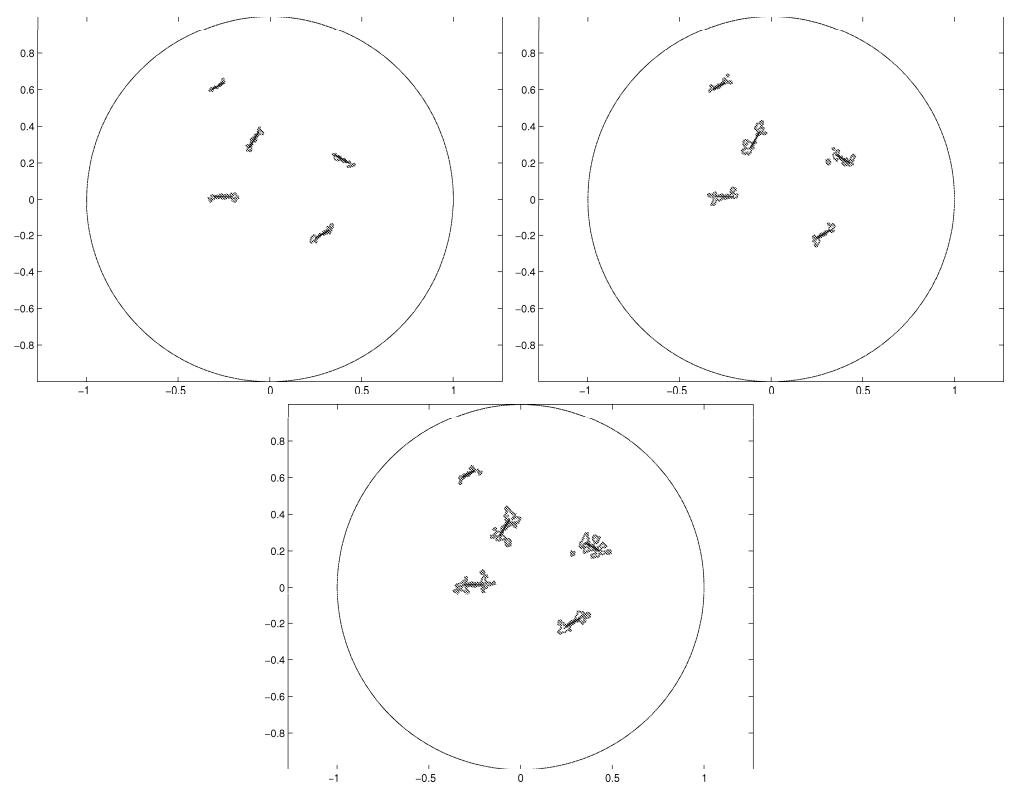

Figure 5. Isovalues of the topological gradient. Noise: 5\% (left top), 10\% (right top), and 20\% (down) (courtesy S.Amstutz, I.Horchani).

nal report, n. 555, CMAP, Ecole Polytechnique

[AmK04] H.Ammari and H.Kang Reconstruction of small inhomogeneities from Boundary Measurements, Lecture Notes in Math. 1846 (Springer), 2004.

[AVV01] H.Ammari, M.Vogelius and D.Volkov, Asymptotic formulas for perturbation in the electromagnetic fields due to the presence of inhomogeneities of small diameter II.The full Maxwell equations, J.Math Pures et Appl 80, 769-814 (2001).

[AVV03] H.Ammari, M.Vogelius and D.Volkov, Boundary ntegral formulas for the reconstruction of electromagnetic imperfections of small diameter, ESAIM:COCV 9, 49-66 (2003).

[Ams03] S.Amstutz Aspects théoriques et numériques en optimisation de forme topologique, thèse de doctorat de l'INSA, 2003.

[Ams05] S.Amstutz Topological sensitivity analysis for some nonlinear PDE systems, to appear J.Math.Pures Appl.

[AmA05] S.Amstutz, H.Andra A new algorithm for topology optimization using a level-set method, preprint http://www.itwm.fraunhofer.de/en/zentral__berichte/berichte/

[ADS04] S. Amstutz, N. Dominguez, B. Samet, Sensitivity analysis with respect to the insertion of small inhomogeneities, proceedings of ECCOMAS 2004. http://www.imamod.ru/ serge/arc/conf/ECCOMAS_2004/ECCOMAS_V2/proceedings/pdf/560.pdf 
[AHM05] S.Amstutz, I.Horchani and M.Masmoudi Crack detection by the topological gradient method Control and Cybernetics. Vol 34, n¹, pp. 119-138, 2005.

[AnB96] S. Andrieux and A. Ben Abda. Identification of planar cracks by complete overdetermined data: inversion formulae. Inverse Problem 12, 553-563, 1996.

[Ben96] M. Bendsoe. Optimal topology design of continuum structure: an introduction. Technical report, Departement of mathematics, Technical University of Denmark, DK2800 Lyngby, Denmark, september 1996.

[Bre83] H. Brézis Analyse fonctionnelle, théorie et applications, éd. Masson (1983)

[BHR04] M.Burger, B.Hackl, W.Ring, Incorporating topological derivatives into levelset methods J.Comp.Phys. 194/1 344-362, 2004.

[CGM73] J.Céa, A.Gioan, J.Michel Quelques résultats sur l'identification de domaines CALCOLO 1973.

[Cea86] J.Céa Conception optimale ou identification de forme, calcul rapide de la dérivée directionnelle de la fonction coût MAAN, 20(3), 371-402 (1986).

[EsS94] H.Eschenauer and A.Schumacher Bubble method for topology and shape optimization of structures. Struct.Optim. 8, pp.42-51, 1994.

[FrV89] A. Friedman and M.S. Vogelius. Determining cracks by boundary measurements, Indiana Univ. Math. J. 38(3), 527-556, 1989.

[GGM01] S. Garreau, Ph. Guillaume and M. Masmoudi. The topological asymptotic for PDE systems: the elasticity case, SIAM J. Control. Optim., 39(6), 1756-1778, 2001.

[GuS01] Ph. Guillaume, K. Sididris. Topological sensitivity and shape optimization for the Stokes equations, Rapport MIP no.01-24, 2001.

[HaM04] M. Hassine, M. Masmoudi. The topological asymptotic expansion for the Quasi-Stokes problem, ESAIM Control Optim. Calc. Var. 10, n 4, pp. 478-504, 2004.

[Ili92] A.M.Il'in Matching of Asymptotic Expansions of Solutions of Boundary value Problems, Translations of Mathematical Monographs vol.102 (AMS), 1992.

[Jao77] M. Jaoua. Equations intégrales pour un problème singulier dans le plan. Thèse, Université Pierre et Marie Curie, 1977.

[KaS97] C.Kane and M.Schoenauer, Optimisation topologique de formes par algorithmes génétiques, Revue Française de Mécanique, 4 237-246, 1997.

[KoV87] R. Kohn, M. Vogelius. Relaxation of a variational method for impedance computed tomography. Comm. Pure Appl. Math. 40(6), 745-777, 1987.

[Lio76] J.-L. Lions Sur quelques questions d'analyse, de mécanique et de contrôle optimal. Les Presses de l'Université de Montréal, 1976.

[Mas02] M. Masmoudi. The Toplogical Asymptotic, in Computational Methods for Control Applications. International Series GAKUTO, 2002.

[MPS05] M.Masmoudi, J.Pommier, B.Samet The topological asymptotic expansion for the Maxwell equation and some applications Inverse Problems 21 547-564, 2005.

[MNP00] V. Maz'ya, S. Nazarov and B. Plamenevskij. Asymptotic theory of elliptic boundary value problems in singularly perturbed domains. Operator theory, advances and applications, 
Vol. 101, Birkhäuser Verlag, 2000.

[SAM03] B. Samet, S. Amstutz and M. Masmoudi. The topological asymptotic for the Helmholtz equation. SIAM J. Control. Optim. 42(5), 1523-1544, 2003.

[San96] F.Santosa A level set approach for inverse problems involving obstacles ESAIM-COCV 1 17-33, 1996.

[SKJ96] M.Schoenauer, L.Kallel and F.Jouve Mechanical inclusions identification by evolutionnary computation, Revue Européenne des Eléments Finis 5 619-648, 1996.

[Sch95] A. Schumacher. Topologieoptimisierung von Bauteilstrukturen unter Verwendung von Lopchpositionierungkrieterien. Thesis, Universität-Gesamthochschule-Siegen, 1995.

[SoZ99] J. Sokolowski and A. Zochowski. On the topological derivative in shape optimization. SIAM J. Control Optim. 37, 1241-1272, 1999.

[WYW04] X.Wang, M.Yulin, M.Wang, Incorporating topological derivatives into lele set methods for structural topology optimization, in Optimal shape design and modeling, T.Lewinski et al.eds., 145-157, Polish academy of Sciences, Warsaw, 2004. 